\title{
SATReLO: A Tool To Support Language Therapies For Children With Hearing Disabilities Using Video Games
}

\author{
SATReLO: una herramienta para apoyar las terapias del lenguaje en niños con discapacidad
} auditiva

Juan Carlos Martínez Arias (D) 1*, Gloria Inés Álvarez Vargas (D) ${ }^{1}$, Martín Vladimir Alonso Sierra Galvis María Constanza Pabón (D) 1 , Diego Linares (D) ', Andrés Darío Castillo (D)2 , Anita Yolanda Portilla (D)', Valeria Almanza (iD) 2

${ }^{1}$ Grupo de Investigación Destino, Facultad de Ingeniería y Ciencias, Pontificia Universidad Javeriana de Cali. Calle 18 \# 118-250. C. P. 760031. Cali, Colombia.

${ }^{2}$ Instituto para Niños Ciegos y Sordos del Valle del Cauca. Carrera 38 \# 5B1-39. C.P. 760042. Cali, Colombia.

\section{CITE THIS ARTICLE AS:}

J. C. Martínez, G. Álvarez, M. V.

A. Sierra, M. C. Pabón, D. L.

Linares, A. D. Castillo, A. Y.

Portilla and V. Almanza.

"SATReLO: A Tool To Support

Language Therapies For

Children With Hearing

Disabilities Using Video

Games", Revista Facultad de

Ingeniería Universidad de

Antioquia, no. 99, pp. 99-112, Apr-Jun 2021. [Online].

Available: https :

//www.doi.org/10.17533/

udea.redin. 20200586

\section{ARTICLE INFO:}

Received: October 22, 2019

Accepted: June 4, 2020

Available online: June 4, 2020

\section{KEYWORDS:}

Video games; speech therapy; educational games

Videojuego; ortofonía; juego educativo

\begin{abstract}
SATReLO is a tool for the creation of customized applications that support language therapy for children with hearing disabilities. These applications consist of video games that replicate therapeutic activities. Video games can motivate children to embrace therapy positively, increasing the time they dedicate towards this therapy, especially at home. SATReLO allows therapists to customize video games according to the needs of each patient and it keeps a record of his or her progress over time. SATReLO contains a software product line, which makes it possible to derive new video games in real time. The process of testing the system, both in terms of functionality and usability, was thorough and allowed many details of its operation to be fine-tuned. Preliminary tests about the impact of the video games in therapeutic process have been very positive.
\end{abstract}

RESUMEN: SATReLO es una herramienta para la generación de aplicaciones personalizadas que apoyan la terapia del lenguaje en niños con discapacidad auditiva. Estas aplicaciones consisten en videojuegos que reproducen actividades terapéuticas. Los videojuegos pueden motivar a los niños a aceptar la terapia de forma positiva, aumentando el tiempo que le dedican, especialmente en casa. SATReLO permite a los terapeutas personalizar videojuegos según las necesidades de cada paciente, y lleva un registro de su evolución a lo largo del tiempo. SATReLO contiene una línea de productos de software, que permite derivar los videojuegos en tiempo real. El proceso de pruebas del sistema, tanto en el aspecto funcional como de usabilidad, fue riguroso y permitió afinar muchos detalles de su funcionamiento. Los resultados de las pruebas preliminares sobre el impacto de los videojuegos en el proceso terapéutico han sido muy positivos.

\section{Introduction}

During the last decade, video games have been consolidating as tools to support rehabilitation processes. Some of the reasons that consolidated the use of video games in this context are: video games make some

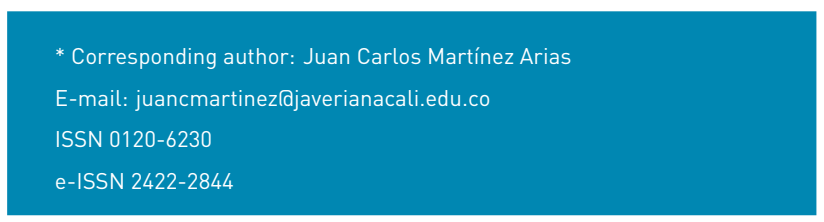

repetitive or mechanical tasks more entertaining, especially for children; patients are more motivated to carry out activities related to their therapies more regularly and patients are better able to understand their therapeutic process in a more positive way. Evidence for these reasons are the positive results reported in previous studies into the development of social skills [1] as well as cognitive and learning development in specific areas of knowledge [2]. Additionally, video games can improve patients' attention and concentration [3], as 
shown by the examples citing the use of video games in the rehabilitation of cerebral palsy and autism.

Previous studies in the area of language therapy, [4-7], have shown the potential of video games as a useful complement, stimulating children to improve their skills by performing their therapy activities more frequently. However, an important limitation to the use of video games in language therapies is the lack of options to customize the games to the needs of each child [8]. One of the purposes of this project has been to address this limitation.

SATReLO is a tool for supporting language rehabilitation processes for oralized children with hearing impairments, allowing the therapist to customize video games according to the specific needs of each patient. This provides desirable effects in patient motivation at both therapeutic and educational levels.

SATReLO's video games support the development of several aspects of oral and written language, particularly for children's narrative skills. The video games were designed by an interdisciplinary team that includes professionals in language therapy, psychology, neuropsychology, software development, and graphic design.

The activities were carefully designed and took into account therapeutic considerations. SATReLO provides tools to assess the language constructions used by patients during the execution of activities in such a way that the tool allows therapists to monitor the progress of the development of the patient's skills.

One of the main characteristics of SATReLO is that the therapist generates customized games according to the current needs of each patient. To this end, SATReLO includes a software product line (SPL). SPLs offer a solution to achieve a balance between offering personalized products and low-cost customization through the systematic reuse of software components.

Two usability evaluations have been carried out. The first made it possible to improve the video games that were developed. The results of the second evaluation showed that the application was ready to evaluate the impact of the tool when used as part of a therapeutic process.

The purpose of this article is to present technical aspects of the development of SATReLO. Following this approach, the remainder of this paper is organized as follows: Section 2 presents the works related to our proposal. Section 3 introduces the problem that SATReLO aims to contribute and the context in which the tool will be used. Section 4 presents the methodological aspects of the development of SATReLO. Section 5 describes the tool. In Section 6, the usability evaluation, and its results are presented. Finally, Section 7 presents conclusions and future work.

\section{Related work}

Other systems have previously been developed that use video games as a strategy to contribute to language development in children with hearing loss. The Speech Viewer [9] was possibly the first system involving video games in an oral therapy support system. In this video game, the correct pronunciation of a vowel made a monkey climb a tree. Later, a game inspired by Pac-Man was proposed [10]. This game associated the vocalization of the name related to each ghost with the attack made by the main character on each one. However, this game had difficulties and delays in vocalization recognition. Talking to Teo video game [4] implements game levels based on simple tasks, similar to those proposed in Speech Viewer, but organized within a more complex narrative. This project demonstrated the contribution of video games to an increase in children's involvement and enjoyment. The games encouraged patients to participate far more in these activities and to repeat them.

On the other hand, in [11] developed Intense, a game for mobile devices, specifically the iPad. The main objective of this game is to support language therapy for children. It focuses on the development of language skills, especially verbal tenses. In [12] authors offer a comparison of different technologies focused on speech therapies, which take into account whether the technology has speech recognition or compatibility with mobile devices. Based on this study, and with the collaboration of experts, they created a speech recognition system and two prototypes that use it: Speech Adventure and Speech with Sam. These prototypes are the basis for Spokelt, a comic book style mini-game aimed at supporting speech therapies.

Other authors [13] have evidenced the usefulness of the mobile phone version of these types of therapy support games. In this work, the patient receives feedback about vowels pronounced through Arcade games. Additionally there are systems that encourage the development of cognitive skills in children with hearing disabilities. The Playmat [14] is a sensor-based system for improving motor and cognitive skills through the recognition of vibrational patterns and textures. Another system [15] trains spatial skills using video games. However, these systems are not focused on the development of cognitive abilities in order to improve the language. 
Regarding the use of the Software Product Lines for the generation of games, a Product Line was used with a component-based architecture, to build video games customized to different platforms such as PlayStation, XBOX, PC, and other mobile devices [16]. Other authors [17] shows a study related to the implementation of artifacts or assets following the Software Product Lines paradigm. They analyze three mobile video games to generate a fourth video game from the artifacts resulting from that analysis. Likewise, [18] summarize the reuse that can occur in the development of video games. This is an important aspect of the Software Product Lines, since reuse is one of the most outstanding concepts in this area. Finally, Software Product Lines have been used in $[6,7]$ to generate customized activities that support the development of various language skills in children with hearing disabilities. These proposals have demonstrated the potential of customized video games as supports for language therapies.

As noted above, there are studies about software focused on language rehabilitation and video games created from Software Product Lines. One of the factors that differentiates SATReLO, lies in the possibility of creating activities (such as video games) customized according to the particular needs of patients. In addition, they are activities that are ready to be used immediately after their creation, without the intervention of software developers.

\section{Context}

The design of specific language therapy to develop oral language in children with hearing loss begins with the acquisition of the vocabulary needed to make semantic descriptions. A semantic description expresses reality by describing the objects, places, environments, people, processes and concepts that comprise it. The description that focuses on aspects explicitly presented is called a static description. However, a dynamic description requires using abstract thinking to connect events, inferring/deducing elements that do not appear in the scene to be described. Children with hearing loss who are oralized, develop less abstract thinking, making it essential to find ways to support them in the development of their description skills. According to previous studies [4], and the concepts given by therapists from the Instituto para Niños Ciegos y Sordos del Valle del Cauca (INCS), customized video games could complement therapy in a positive way. These video games are therapeutic activities previously designed by an interdisciplinary team that includes language therapists and psychologists.

Furthermore, children with hearing loss require a rehabilitation process in order to acquire communicative skills in all dimensions of language. When using the oral method, children may find difficulty in syntactic structuring and the development of the oral and written narrative. Therefore, rehabilitation must allow children with hearing loss to develop language at a level as close to that of hearing children as possible.

When working with deaf children who are users of hearing aids (for example, cochlear implants), it is important to promote the development of the following aspects: listening, speaking, thinking, remembering, understanding, comparing, sequencing, and temporal relationships. Further characteristics to be developed are cause-effect relationships and the acquisition and development of syntax and executive functions. These characteristics are altered in patients with this type of impairment, as shown by Soriano in his comparative study on the narrative of deaf and hearing school children [19].

At the INCS, different strategies are used in order to achieve the development of oral language in children with hearing loss. The therapeutic activities materialize those strategies to favor the development of oral and written language. The different levels of language and thinking skills are taken into account when generating activities geared towards the facilitation and visualization of thought and the speed at which children process information. The activities initially proposed for implementation in this project were a domino game, a tidying up the house game and a sequence cartoon:

- The Domino game favors the development of basic elements of language for static description. Based on syntax, it allows for the structuring of simple sentences while taking into account different grammatical elements. The game favors the nomination of different semantic categories, thus allowing for further comparisons. At the level of thought or cognition, it stimulates the ability to make observations so that the child determines the physical characteristics of the objects that appear in the game. This further promoting the child's ability to comprehend the variables that allow for playing.

- Tidying up the house allows practicing static description. The activity takes place in the context of a child's room that is messy. The child helps the main character by receiving instructions, constructing sentences, and performing activities to tidy up the room. For example, pick up a garment of a certain color and store it in the closet, or to wash a garment. Within this dynamic, the use of pronouns, nouns, verbs, and qualifying adjectives are practiced, organizing them within correct sentences. From the cognitive perspective, the skills of; observation, identification, comparison, denomination, and description are developed. 
- Sequence cartoon is designed for the practice of dynamic descriptions. From the semantic perspective, it favors the children's comprehension skills and their interpretation of events, establishing and organizing a succession of situations that share a certain relationship between them. This activity favors an oral and written expression of events of differing characteristics, developed in a specific time and place, explaining as to why the events occurred in the story.

\section{Challenges}

Among the main challenges for developing these proposed therapeutic activities, this project was focused on five in particular: offering customized therapy activities, generating a final product, helping parents to guide their children when practicing at home, providing therapists with options to observe the progress of children in carrying out activities and motivating children to do their practices.

- Customization: each child has a different biological and mental age, gender, beliefs, attitudes, personality, and psychological characteristics $[20,21]$. Therefore, customized models that take into account the specific needs of each child are key to providing the care required by each child with the aforementioned disabilities.

- Final product: therapists need to customize the activities to be assigned to patients during the therapy session ldepending on the patient's progress and evaluation). This is important because during the therapy, the therapist can familiarize patients and their family with the activities that need to be carried out at home.

- Home-support: parents have a fundamental role in the rehabilitation process. One of the ways families participate in the process is at home, when they help children to repeat the activities that took place during the session with the therapist or to do the homework assigned by the therapist. However, it is difficult for therapists to monitor the effectiveness of exercises done at home. One reason is that families generally do not have enough training to guide these activities on their own. Another reason, is that families do not record the results of each activity.

- Feedback: therapists need information about the execution of the activities carried out by the child, especially at home. It is also necessary to automatically record information regarding the execution of activities. This information must provide the therapist with data about the child's activity, both in terms of the number of exercises and success in performing them.
- Motivation: in many cases, children show a lack of motivation in carrying out repetitive tasks. This may affect children's ability to achieve therapeutic goals.

SATReLO is a tool that responds to these challenges and consists of a software product line of custom-made video games and two web portals that facilitate the interaction of therapists and patients with the software product line.

\section{Development method}

SATReLO was developed by combining a software product line development methodology, with a user-centered development approach, to ensure not only the usability of the system but also that the activities respond to the therapeutic objectives for which they were designed.

\subsection{Software product line development framework}

Software Product Line Engineering (SPLE) is a production paradigm that enables efficient development and management of products with common and variable elements, by reusing those elements [22]. Therefore, a Software Product Line (SPL) makes it easier and faster to generate customized products [22]. The common elements, also called core assets, can be, among other things, source code, documentation, or architectural documents [23].

For the construction of the SPL of video games, we followed the methodology proposed in [24], which specifies two principal stages: domain engineering and application engineering.

Domain engineering comprises the domain analysis and domain implementation. The first includes; an analysis of the application area of the products in the line, the definition of the SPL scope and the identification of common and variable elements. The feature model is a notation widely adopted in the SPL community to capture the product line commonality and variability [25]. The domain implementation consists of the design and construction of the core assets that support the product line [22].

Application engineering is the stage at which products are built. It consists of a product configuration phase and an assembly phase. In the configuration, users select, in a guided way, the functional features that best suit their needs [22]. A valid configuration not only satisfies the user's requirements but also the restrictions of the SPL domain and other non-functional characteristics [26]. Moreover, the features selected should not conflict with each other. 
Once the products are configured, the software assets that implement the features selected are assembled to deliver a software product [22]. Thus, in the assembly phase, a final product of the SPL is generated automatically or semi-automatically.

The development of tools and methods to support configuration and assembly is still an open field of research. It is worth noting that one of the results of this project is a system that automates the configuration and assembly stages of our SPL of video games.

\subsection{Interdisciplinary team work}

An interdisciplinary team made up of language therapy and software development professionals worked on all stages of development of the product line. The team evaluated each design decision; relating to user interfaces, software product line and the implementation of core assets and web portals, according to their impact on the therapeutic purposes of each activity.

Section 5.1 gives details on how the team approached each stage of the development of SATReLO.

\subsection{Usability aspects}

In SATReLO, usability is a key element to achieve the greatest positive impact. For this reason, the design of the interaction between users and the system was central to the project, and all 10 Nielsen heuristics [27][28] were taken into account. The specific aspects of the system in which these recommendations are addressed are described below:

1. Visibility of the system status: A basic feature of the system is the feedback given to users at each step of the development of their activities. Beyond this, SATReLO video games share a homogeneous interaction style. An avatar gives children clear and precise instructions about the activity they are doing. Likewise, the points accumulated in the activity are visible at all times.

2. Match between the system and the real world: The avatar is the representation of the therapist in the system; it is configurable to look like the real therapist (e.g., sex, hair color). Moreover, the video game follows the same interaction scheme as the interaction with the therapist during a face-to-face session. Additionally, video games also resemble the activities that children do in their face-to-face therapy sessions.

3. User control and freedom: Video games have an exit button to end the activity at any time. However, during usability testing, the need to provide additional controls to strengthen this aspect was identified.

4. Consistency and standards: SATReLO games use the same icons to turn the sound on or off, display the score, and end the activity. They also run from the same frame, the patient portal, giving uniform access to the activities. The avatar is one element that unifies the way children interact with the game. There is also consistency in the terms used to refer to the common elements of the games.

5. Error prevention: Unlike other types of systems, it is not a goal of video games to avoid mistakes, especially in the context of games that support skill development. Outside the game dynamics, and within the possible, the system prevents error-prone actions. For example, the system detects when the Internet connection has been lost and stops working until the connection is restored.

6. Recognition rather than recall: During the video game, the avatar is always active, giving instructions that correspond to each stage of the game. In this way, the child does not need to remember aspects related to the interaction in the game. These instructions can be received orally, in written form, or by using both options simultaneously.

7. Flexibility and efficiency of use: The explanations about the general functioning of the system, and particularly those of the video games, can be deactivated in case the child already knows them and wants to go directly to play.

8. Aesthetic and minimalist design: As a requirement of therapists, given the target audience of video games, the interface should be kept simple but appealing, including only the elements necessary for the development of the activity and to achieve the learning objectives. The location of the elements in the interface was made so that it looks clear. Its most relevant elements are highlighted. The aim was to give only the necessary information in each view of the system.

9. Help users recognize, diagnose and recover from errors: By design, the system is prevented from issuing error messages to users, as it is understood that these messages are not useful to them. The few messages that appear have to do with problems connecting to the server.

10. Help and documentation: In addition to the avatar guide, each activity has a user manual and a video tutorial that can be watched before starting to play. The videos are short and show the development of the activity step by step. Additionally, for therapists, there 


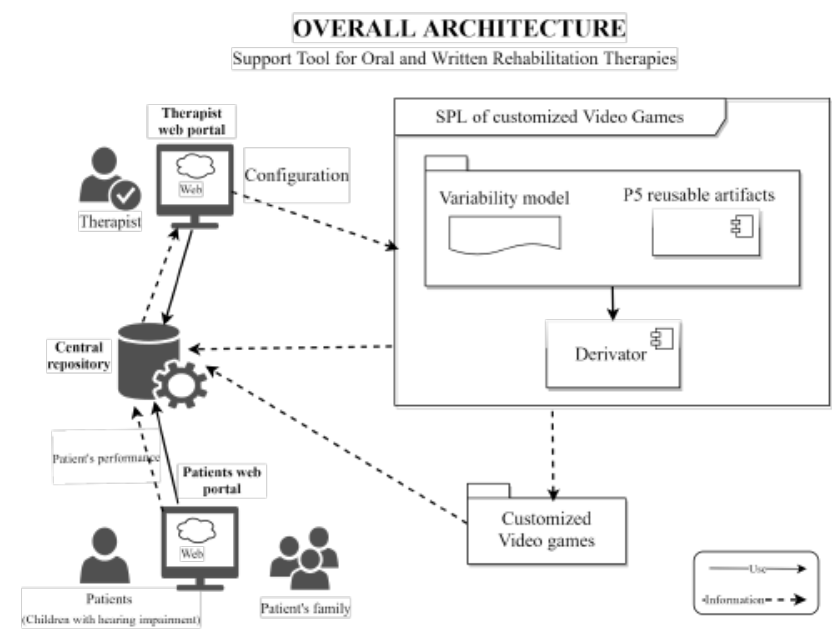

Figure 1 SATReLO overall architecture

is a document that describes the characteristics and contributions of the game from a therapeutic point of view.

\section{The SATReLO tool}

SATReLO is a computational tool that allows the construction of customized video games that support language therapy for oralized children with hearing loss and apply the paradigm of software product lines. Figure 1 shows the overall architecture of the SATReLO.

\subsection{Software product line for therapy activities}

In accordance with the proposed methodology, the following steps were carried out in Domain Engineering and Application Engineering:

\section{Domain engineering}

The scope of the product line for video games was defined in multiple meetings to elicit the requirements of therapists and patients. Those meetings were held among an interdisciplinary team including people with expertise in software engineering, product lines, visual design, and the therapeutic process. We defined a collection of candidate activities that support previously defined requirements. The INCS therapists and members of the project research team carried out tests with patients. These tests were intended to validate whether previously defined activities were useful in achieving therapeutic objectives. The therapists used physical prototypes of the activities to perform this task. Lastly, we built low fidelity prototypes (on paper and mock-up design tools) to determine the application flows and agree on the structure and identify new requirements.

Based on the narrative development process and the requirements of the activities, the team modeled the product line commonality and variability using feature models. The resulting model has 66 features including features related to the structure of the oral language and the mechanics and structure of the activities. Among the various features, the mechanics of the activity defines the game's behavior and rules. The combination of a mechanic with variability options included in the feature model of the product line produces different products, each one focused on different abilities and stages of the narrative development process. The combination of these elements pursues a specific therapeutic objective. Figure 2 shows the first level of SATReLO's feature model; due to space limitations, this document does not show the complete model, but readers can view it on-line (https ://www.researchgate.net/project/Apoyoa-la-Terapia-de-Rehabilitacion-del-LenguajeOral-y-Escrito-en-Ninos-con-DiscapacidadAuditival.

At the implementation level, the reference architecture for the product line and the web portals were defined. The web portals have a layered architecture that hierarchically organizes elements that make up the system. For example; the graphics interface, controllers, derivator and the database. A Model-View-ViewModel (MVVM) software architecture pattern [29] was used for the construction of the SPL reference architecture. With this pattern, it is possible to decouple the graphics interface from the application logic. On the other hand, variability in components was implemented through conditional compilation and passing parameters to condition the execution of the activities.

We have incrementally designed and implemented reusable components that support the customized video games. We also built the data model responsible for storing information about the reusable components, the configuration options and the patient's progress.

So far, we have developed common and variable reusable artifacts to derivate the video games.

\section{Application engineering}

The web portals are the mechanism that SATReLO provides to make interaction easy between therapists and children with regard to the application engineering part of the SPL. The feature selection and product derivation are embedded in the therapist portal, while the execution of the generated products is embedded in both portals.

Therapists make the product configuration las defined in 


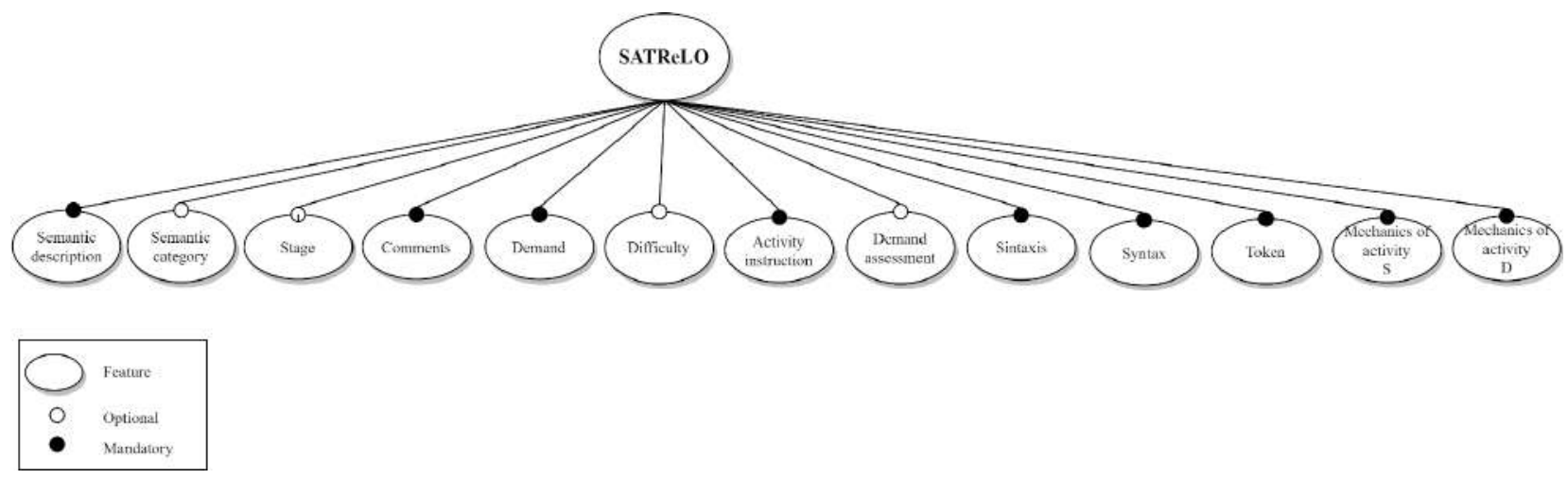

Figure 2 SATRELO's SPL feature model first level

[24]) to generate a customized activity that is assigned to a patient. Information for configuration is saved in a common repository that is consulted to perform the derivation. SATReLO's derivator has the responsibility for assembling and customizing reusable JavaScript library P5 artifacts [30]. The process is fully-automated and its purpose is to create a final customized video game.

The product line of customized video games generates activities with the following features:

- Domain-oriented: explicitly designed to support therapies required by children in hearing impairment rehabilitation.

- Based on reusable components: reusable software artifacts elaborated during the domain engineering process are the basis for producing the customized video games. Those software artifacts were developed considering their potential reuse, which implied an important technical challenge in this project.

- Customized: therapist can choose from a set of predefined options those that better fit the needs of their patients. Then, based on their selections the customized video games are derived. To ease this configuration process, a web portal for therapists was created.

- Final: resulting video games do not need any extra intervention to be a completely functional software unit. However, to ease the access of patients to those activities, a web portal just for patients was created.

\subsection{SATReLO platform}

SATReLO is made up of three main modules. The therapist's portal, where the therapist assigns the activities to be carried out by the child and customizes them. The child's portal, where the child performs the activities assigned by the therapist. The video game generator. Figure 3 describes SATReLO's general concept.

Regarding the implementation of the system, the portals of the therapist and the child were developed in web languages, like Html, CSS, and Javascript. The library react was used for the creation of the visual components of the interface of the sites. The components for the video games were developed in the P5 library of javascript [31]. The server, where the source code and the database are stored, was built using the multiplatform Node JS environment. The database was built in PostgreSQL.

The therapist's portal allows for: the managing of the patients; configuring, generating and assigning video games to a given patient; and the monitoring of the patient's progress.

According to the configuration that the therapist selects, the components needed to build the game are taken from a data store and assembled to build the desired activity. For example, the therapist can select the language attributes that the video game will contain: articles, pronouns, nouns, adjectives, and verbs. $\mathrm{He} / \mathrm{she}$ can also select the theme. The options available in this version are: animals, school supplies, and professions. The rest of the customization options are selected analogously.

The patient's portal allows the child to use the video games assigned by the therapist. Figure 4 shows the initial screen.

The portal has a support avatar which represents the therapist in this virtual environment. This virtual therapist gives work instructions similar to those given by a real therapist in live sessions that take place at the INCS. For example, in the case of Domino: "join and match similar elements". Another example, can be seen in the Sequence cartoon: "organize a series of images in a logical way to build a story". The avatar responds in real-time to the patient's interactions and to events that occur in the 


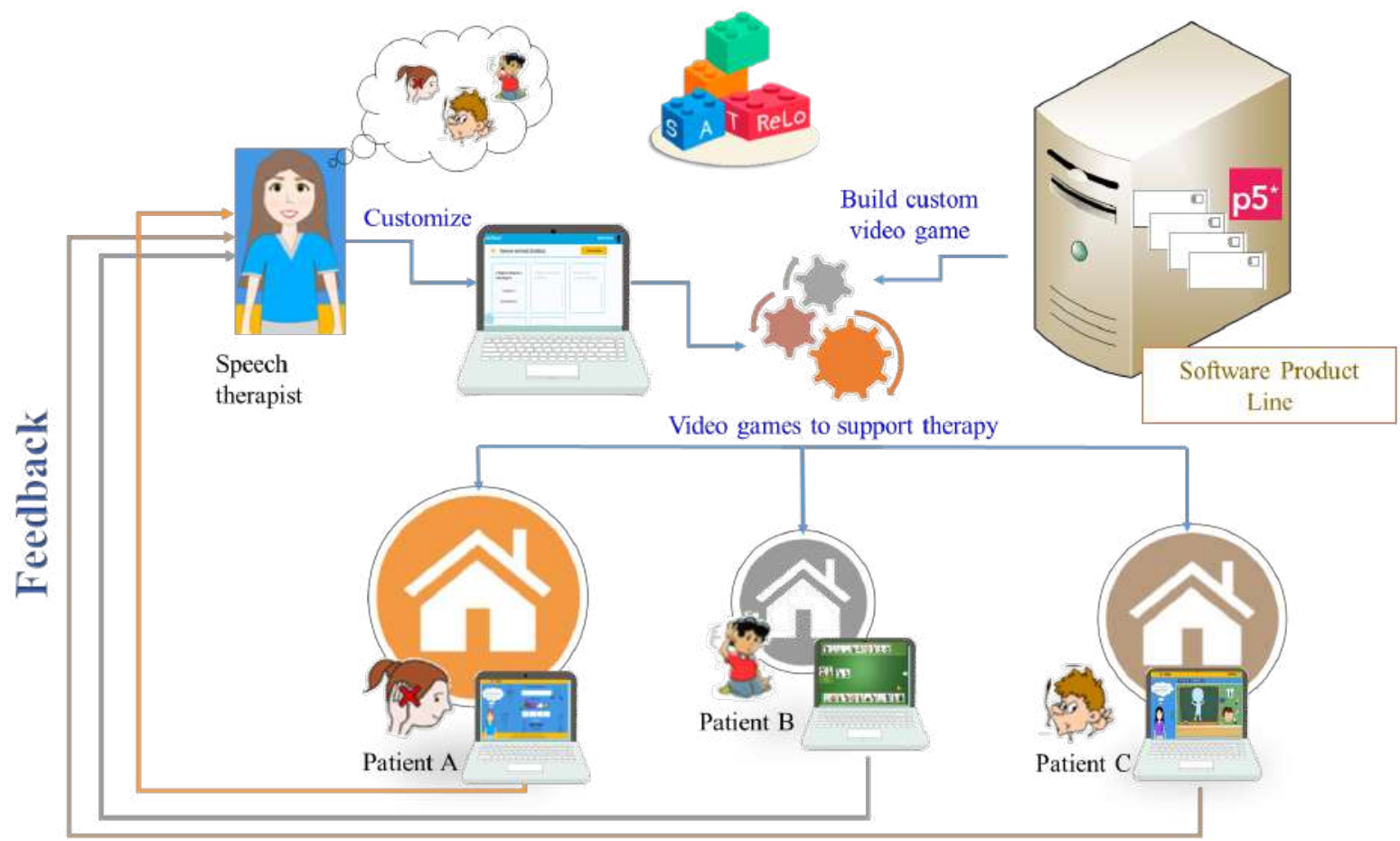

Figure 3 Interaction among SATReLO, therapists and patients

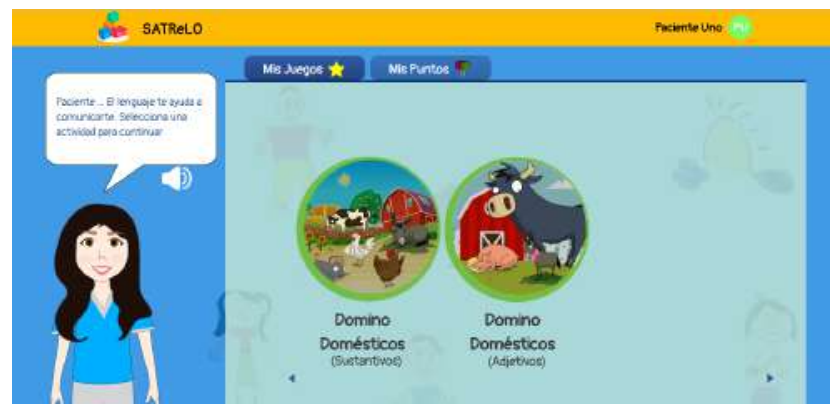

Figure 4 Main view of patient's portal

video game, by either completing an action correctly or incorrectly.

\subsection{The video games}

This section describes the video games that can be generated by SATReLO.

\section{Domino}

Domino, is a video game that favors the development of static description. It consists mainly of matching tiles of the same type. The correct pairing of cards depends on the configuration made by the therapist in their portal. For example, in the case of domestic animals, if the configuration is set up for nouns, the patient will need to match hens with hens, cats with cats, dogs with dogs. If the configuration is oriented to adjectives, the patient will need to match a beautiful animal with a beautiful animal, a fat animal with a fat animal and so on. The activity is also developed in the same way for the configuration of verbs. Figure 5 shows the Domino video game running in the patient's portal.

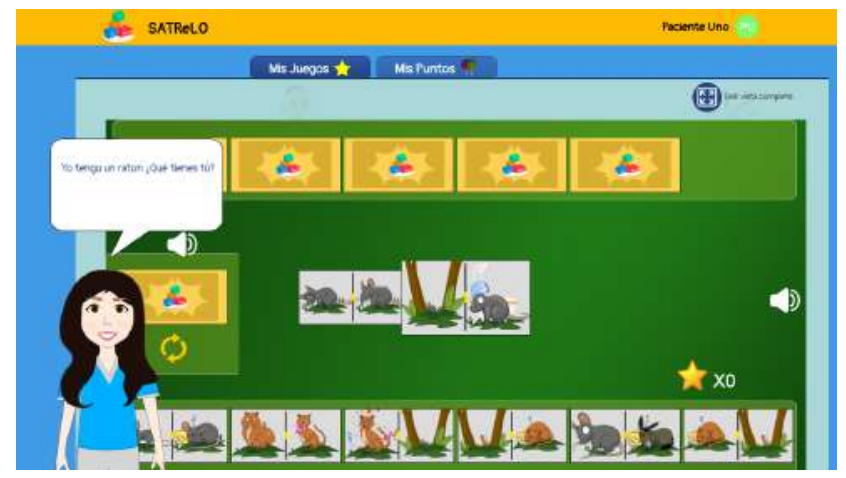

Figure 5 Video game domino

The game begins with a starting piece placed on the board. Both the avatar and the child have their own set of cards to play with llocated at the top and bottom of the screen, respectively). The first turn is for the avatar, to show the child how to play. Thereafter the turns alternate. When a child's move is correct, he or she obtains some points. Otherwise, if the pairing made by the child is incorrect, the 
card returns to its place of origin. If at any time the child does not have a matching card, the game gives him or her the opportunity to take a new one.

\section{Tidying up the house}

Tidying up the house, is a video game that develops children's static descriptions, the same way Domino does. Articles, nouns, adjectives, verbs, and conjunctions are worked on in this video game. Figure 6 shows the video game running in the patient's portal.

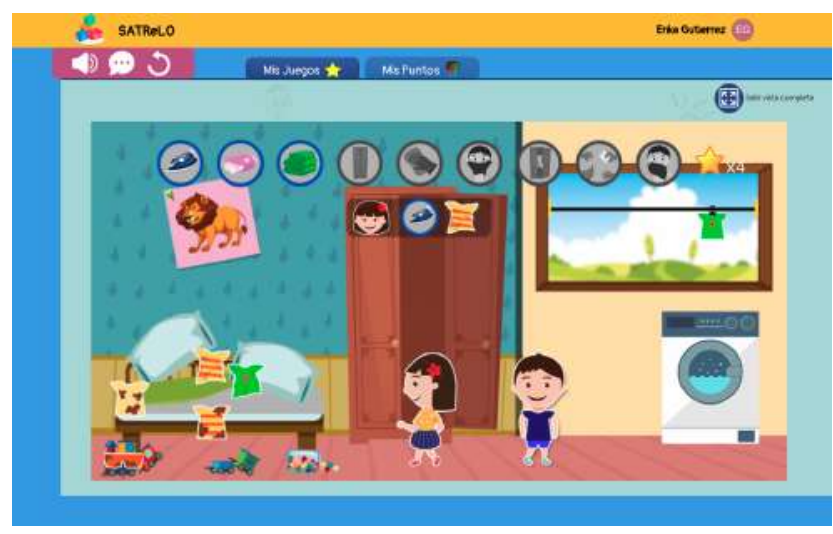

Figure 6 Video game tidying up the house

The video game consists of two parts:

- The first part of the activity presents the child with a narrative that places him in the context in which the game will take place. In this part, the child answers questions related to the images that the game shows him.

- The second part places the child in a room in the house where the child begins to play following the instructions given by the avatar.

The video game shows a boy and a girl in a messy room, where there are clothes with different characteristics. Depending on the characteristic of the clothes, the avatar gives instructions for the child to perform. For example, for a dirty shirt, the avatar says: "Move Sofia to wash the dirty shirt." For this instruction, the player will need to first select the girl, then select the image that represents the verb wash (located at the top of the screen). Finally, the child must select the shirt. If the sequence of selections is correct, the player wins points, and a small animation appears where the girl takes the shirt and takes it to the washing machine. The activity ends once all the clothes have been tidied up. As in all the SATReLO's video games, the clothes and the verbs that are shown in the activity, were previously customized by the therapist.

\section{Sequence cartoon}

This video game integrates activities associated with dynamic descriptions. The activity is composed of seven different levels. Each level increases in difficulty and allows the child to work on different skills, such as organizing sequences in a logical way, constructing simple and compound sentences, and making inferences. This allows the child to develop more structured stories [32]. Figure 7 shows level one of the video game.

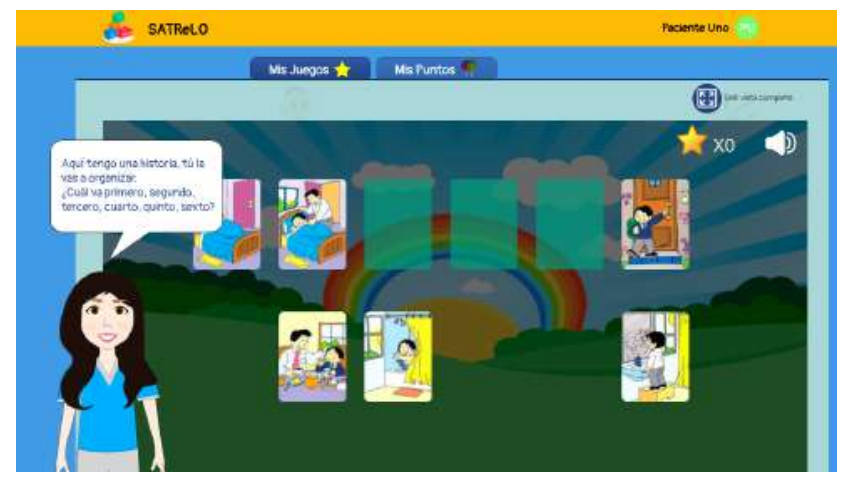

Figure 7 Video game sequential cartoon

The seven levels of the Video game are described below:

- Level 1: The activity presents the child with a set of images that are in no particular order. The objective of the activity is to order the images, so that a logical sequence is constructed as a story.

- Level 2: At this level, two images are presented randomly. The goal in this case is to locate these images in the correct order.

- Level 3: In this part of the activity, an image is randomly selected and removed from the sequence, placing a question mark in its place. On the other hand, the game presents a new type of image called pictographs. The objective is to select the pictograph that relates to the image hidden in the sequence.

- Level 4: At this level, the video game adds a question mark before and after the sequence of images. The objective is to infer what happened before and what happened after in the story, using the pictographs.

- Level 5: This level presents the sequence images with related questions. The goal is for the child to answer these questions with structured sentences.

- Level 6: In this level, the ordered sequence and a text box are presented in which the child must write the whole story.

- Level 7: This last level shows an image and a question related to that image. The goal is for the child to order the words to form the answer to the asked question. 


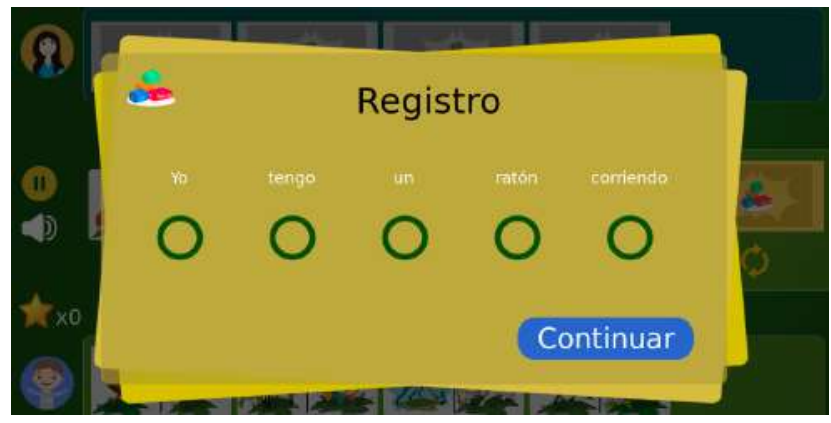

Figure 8 Validation screen in the domino game

\subsection{Answer assessment}

In order to record the actions and verbal expressions performed by the child, after each turn, the video game presents a validation screen. This screen is a floating window that the video game shows for the person accompanying the child to record what the child says.

Activities related to static descriptions (Domino and Tidying up the housel often ask the child for oral answers to an avatar question, associated with the action that has just occurred. Each word that makes up the phrase comes next to a check-box. The person accompanying the child should select the check-box of the words that the child said well. If the child said a wrong word, the checkbox of that word is left unselected. This information will be used to feedback the therapist on the evolution of each child.

In the case of video game, Sequence cartoon, the validation screen is used to record if the patient completed the level alone, with help, or if they definitely didn't do it.

Figure 8 shows an example of the validation screen in the Domino game, while Figure 9 shows an example of how the validation screen looks in the Sequence Cartoon game.

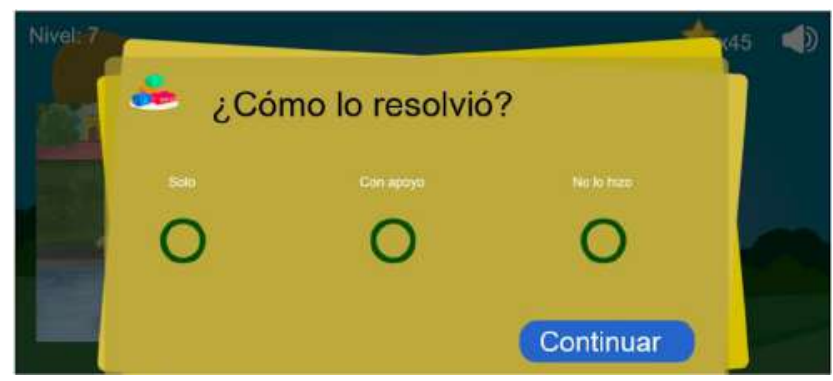

Figure 9 Validation screen in the sequence cartoon game

\subsection{Feedback for the therapist}

To observe children's progress carrying out activities, we designed two options: Progress and History. Progress presents the results the child obtains carrying out activities in a session. History compiles the results that the child obtains during a certain period of time carrying out the activities in the video games. Each of the results can be seen using charts.

In SATReLO, evolution and history concepts were implemented as follows:

- Each time a child starts the execution of a video game, a session is created, and the start and finish time are stored.

- Each turn made by the child, whether right or wrong, is recorded in the database.

For Domino, both types of matches are registered.

Tidying up the house also records right and wrong choices.

For Sequence, correct and incorrect tidy ups are recorded at levels 1, 2, and 7; correct choices at levels 3 and 4; correct and incorrect answers at level 5; and level 6 , records the text that the child wrote.

- The results that the person accompanying the child indicates in the validation screen are registered on the database.

\section{Evaluation of the SATReLO tool}

\subsection{Functional test}

Testing a software product line, such as the one developed in the project described in this article, has its own challenges.

Owing to the number of products that can be generated by an SPL, and because many of these products may share similar characteristics, the amount of testing that must be done becomes unmanageable [33]. That is why testing Software Product Lines should avoid the exhaustive task of testing each product individually. With this in mind, SPLs require testing that meets two criteria: efficiency and effectiveness. Effectiveness refers to the ability of the test cases to reveal as many defects as possible, while efficiency tries to keep the amount of resources used to execute the test to a minimum [33].

Therefore, the components that were reused in the different video games generated by SATReLO, had to be strictly tested (using unit tests), because any component that has a defect can cause failures in other video games that are generated. In turn, some tests can only be 
performed when these components are reused in the different video games lusing integration tests). Under these constraints, a specific test plan was developed based on two criteria: the selection of the most frequently used components and the selection of the most critical components. To apply these criteria, a test model was used that combined two strategies: division of responsibilities and application of samples [34, 35].

For the division of responsibilities, the model proposes that the test levels (unit tests, integration tests, system tests, acceptance tests) be divided into the Domain Engineering and Application Engineering stages. Thus, each reusable component of the SPL should be tested in order to verify that each one meets the requirements in an appropriate manner. These tests were conducted as soon as each component was completed. The unit tests were performed by the working group that participated in the development of the components.

On the other hand, it became necessary to use samples of the video games that could be generated from the SPL. These samples do not correspond specifically to the video games that the application generates for a particular patient, but it is a video game that could be generated at any point in time. The strategy that was used to obtain the sample, was to generate a set of video games that used SPL components that produced a critical situation and that affected a high number of the system's functionalities. A critical situation is understood to be when; there are components that do not working correctly, could lead to a large number of failures in various applications, or if integration with them presents problems.

We performed the following steps to build and run the test cases:

- We performed unit tests on each of the components that make up the SPL, during domain engineering.

- We established the criteria to use for the selection of components for the sample. Two criteria were used: most frequently used components and most critical components.

- We selected the components using the criteria defined above.

- We generated sample video games using the components that were selected in the previous step.

- We performed the test cases for the generated video games.

- We analyzed the results obtained after applying the test cases.
In addition to the test cases made to the SATReLO SPL, traditional tests were made to the platform's two web portals.

\section{Web portal testing}

The design and execution of the test cases was carried out by an expert in the area and independent of the project. 26 test cases were designed for the therapist portal and 13 for the patient portal. For the two portals, 5 iterations were executed in the course of one month. The tests detected minor, moderate and severe failures. Some observations on these failures were:

- Minor: "The portal reports that the data is incorrect, however, this warning disappears a few seconds after it is displayed (it should remain on the screen)".

- Moderated: "When you first enter the modify avatar tab, the avatar appears empty until you click the man or woman button".

- Serious: When you enter a collection (clothes or cars), the system takes too long to place a comment (such as when you finish an activityl. Only when you click again on my points tab, it takes the patient to the collection".

At the end of the five iterations the above problems were resolved.

\section{Video games testing}

Following the proposed strategy (application of samples), the tests were carried out for three different configurations (samples) for each proposed video game (Domino, Tidying up the house and Sequences cartoon). For each video game, five iterations were made, varying the configurations in each iteration, in order to cover as many products as possible.

In the case of Domino, 21 test cases were designed and executed, for Sequences cartoon, 44 test cases were designed and executed, and for Tidying up the house 32. The results of the tests found important elements that made it possible to adjust the operation of the video games. At the end of the five iterations, the different problems were resolved.

\subsection{Usability test}

The purpose of this evaluation phase is to analyze the usability of the customized applications generated by SATReLO. First the structure and characteristics of the test are described and then the results and their analysis are presented with respect to three criteria: effectiveness, efficiency and satisfaction. Since the tests involve children, their parents were told what the test would consist of. They 
gave their authorization for their children's participation and signed the corresponding consent forms. The children were also told what the test consisted of. They were asked if they wanted to participate and they also signed their own consent forms.

The first usability test was performed with a sample of six children. They were INCS patients, aged between five and nine, in transition school, first or second grade. Three of the children in the group used either a hearing aid or cochlear implant as a listening aid. The other three children had normal hearing. The test evaluated the usability of the domino video game: the semantic category was domestic animals and the two customization possibilities in the syntactic structure were nouns and adjectives. The game included 15 animals to be combined and 11 adjectives for each animal. The therapist and the patient sat at the computer, while a test monitor followed the session as an observer, taking notes of the positive and negative aspects identified in the interaction with the tool. The sessions lasted 30 minutes. Afterwards, the children completed their corresponding evaluation questionnaires, in some cases with the aid of a therapist. Questionnaires were prepared using Jakob Nielsen's usability recommendations [27].

Once the results of the questionnaires were tabulated and the observer's session notes were analyzed, the following aspects were identified:

- Effectiveness:

- Children were able to navigate the site, easily selecting a video game and entering into it.

- Children adapted easily, interacting with the application in which the avatar gave them instructions and awaiting their turn in order to perform the corresponding action.

- The operation of the game deteriorates when the internet connection is lost.

- Efficiency:

- The avatar sometimes loses sync between its image and its voice.

- There is a delay in the appearance of the avatar's hair in relation to the appearance of the rest of the body.

- Satisfaction:

- The scheme of the application is that each success in a video game gives points which can then be exchanged for items in order to complete a collection. Children were enthusiastic about this aspect of the application.
- Children complained that the avatar spoke so fast that they had difficulty understanding what was said. In many cases, the therapist had to repeat the instructions so that children could move forward.

- The possibility of exchanging the figures on both sides of the domino card was not easily understood by the children.

- It was not clear to children how to drag a card from the pile in order to continue playing.

Based on the test findings, a new version of the system was developed correcting the problems found, and a second test was applied using a new sample of six children with hearing aids, aged between five and nine years. Four of these children were enrolled in second grade and two of them were enrolled in pre-kindergarten. The test had the same structure as the previous one, and the same questionnaire was given to the children at the end. The main results of the test were the following:

- Effectiveness:

- The therapist can make use of the game both for children who are starting their therapeutic process, as well as those who are more advanced. The game was useful even in the case of children without previous experience using computers.

- The video game helps children practice the association of elements, helps them in their ability to deduce, and helps to stimulate their powers of observation and memory.

- It is important to increase the range in which the volume of the avatar's voice can be set.

- Efficiency:

- It is important that the therapist is able to instruct the game to repeat the last message issued.

- It is important that the game can be paused when the therapist requires.

- Satisfaction:

- Children are active and attentive to the activity; they are interested in continuing to play.

- The child's point count is reset by switching from one activity to another and the points are lost.

Once the adjustments identified in the second test were made, it was decided that the game could be used to begin the evaluation of its impact on the rehabilitation process. During 16 therapy sessions, a group of 12 children, between six and twelve, used the video games, defined 
by their therapists through the SATReLO platform, one or two hours per week. The children were between transition school and third grade, and everybody in the group used either a hearing aid or cochlear implant as a listening aid. These sessions were additional to the usual therapy times. At these practice times, the children worked with the support of a different therapist, who observed the children's interactions with the video games and their therapeutic progress.

Some of the preliminary observations by the therapist who facilitated the video games sessions as a complement to the therapy were:

- By using video games, children make use of everyday expressions and communicative interactions in a natural way, using everyday language according to the game's situation. For example: "The girl is listening", "Calm, calm I go", "Why the girl just looks and looks and does not move, she has no eyes", "Shh ... Do not talk anymore ", Children show interest in communicative interaction through the avatar.

- Children maintained interest and motivation throughout the work activity. One of the most important elements in generating this effect was the interaction with the avatar, which asks questions and makes comments according to the game's situation.

- The activities incorporated into the video games suit different therapeutic goals for different children.

- The video game that children liked the most was Tidying up the house, because it was more interactive or dynamic. The way the characters performed the actions selected by the children was very striking for them.

Given the positive outcome of the preliminary tests into the impact of the SATReLO tool on the therapeutic process of children undergoing speech therapy, it is expected that this evaluation can be deepened in a future project that has a greater therapeutic focus and a less engineering component.

\section{Conclusions}

SATReLO platform is a suitable tool in the field of language rehabilitation, according to the preliminary tests.

The children easily adapted to the interaction protocol in the video games. This could be due to the fact that the same dynamics of the face-to-face therapy sessions were followed and that they had customized activities.

The therapists, on the other hand, highlighted that the tool offered them video games that fit different therapeutic objectives. This maintains children's interest and by achieving that, they will generate greater autonomy in their rehabilitation process and greater oral communicative interaction.

The formation of an interdisciplinary team, combining the efforts of therapists and engineers, has been fundamental for the project, supporting the therapeutic process, while making sure that the project be computationally sound.

Finally, SATReLO is a tool that can be used to make language therapy available even in the most remote places. It can be used as a support tool for tele-rehabilitation programs, allowing more children to benefit from language therapy; providing them with skills to integrate better into society, expanding their possibilities for greater cognitive development.

As technical future work, a greater number of games could be included in the system, in order to give the children more variety of activities to perform their rehabilitation therapies. This inclusion could be provided by the Software Product Line, that supports the game generation system, reusing the components implemented for the games seen in this paper. It may also be possible to devise a way of moving all the work done in the system, which is focused on web environments, to the mobile world. By doing that, the number of children playing the games remotely, to support their rehabilitation therapies with the SATReLO tool, could be higher.

As regards future work on the therapeutic aspects, it will be important to further evaluate the impact of SATReLO on language rehabilitation, as improvements, adjustments and extensions, can emerge from this evaluation to help maximize its positive impact on the process.

\section{Declaration of competing interest}

We declare that we have no significant competing interests including financial or non-financial, professional, or personal interests interfering with the full and objective presentation of the work described in this manuscript.

\section{Acknowledgments}

This research is part of project No.125174455451, funded by the Departamento Administrativo de Ciencia,Tecnología e Innovación de la república de Colombia (COLCIENCIAS), entitled: “Apoyo a la Terapia de Rehabilitación del Lenguaje Oral y Escrito en Niños con Discapacidad Auditiva". 
Likewise, this work has been partially supported by the Pontificia Universidad Javeriana Cali, Colombia and the Instituto para Niños Ciegos y Sordos del Valle del Cauca, Colombia under the project No.1584.

\section{References}

[1] C. Perrotta, G. Featherstone, H. Aston, and E. Houghton, "Game-based learning: Latest evidence and future directions," National Foundation for Educational Research, Slough, England, Tech. Rep., Apr. 2013.

[2] R. Kenny and R. McDaniel, "The role teachers' expectations and value assessments of video games play in their adopting and integrating them into their classrooms," British Journal of Educational Technology, vol. 42, no. 2, March 2011. [Online] Available: https://doi.org/10.1111/j.1467-8535.2009.01007.x

[3] J. Kirriemuir and A. McFarlane, "Literature review in games and learning," Nesta, London, England, Tech. Rep. hal-00190453, 2004.

[4] A. A. Navarro and et al., "Talking to teo : Video game supported speech therapy," Entertainment Computing, vol. 5, no. 4, December 2014. [Online]. Available: https://doi.org/10.1016/j.entcom.2014.10. 005

[5] L. Rincón and et al., “Un videojuego para apoyar la terapia del lenguaje: el caso de la descripción estática," in IX Congreso Iberoamericano de Tecnología de Apoyo a la Discapacidad (Iberdiscap), Bogotá, Colombia, 2017, pp. 597-605.

[6] L. Rincón, J. C. Martínez, M. C. Pabón, J. Mogollón, and A. Caballero, "Creating a software product line of mini-games to support language therapy," in $13^{\text {th }}$ Colombian Conference on Computing, Cartagena, Colombia, 2018, pp. 418-431.

[7] J. C. Martínez and et al., "Using software product lines to support language rehabilitation therapies: An experience report," in ICAI Workshops (ICAIW), 2018, pp. 1-6.

[8] W. R. Rodríguez, "Herramientas informáticas libres para los desórdenes de la comunicación humana," Revista Ciencias de la Salud, vol. 13, no. 2, July 03 2015. [Online]. Available: http: //dx.doi.org/10.12804/revsalud13.02.2015.14

[9] V. Bernard, N. Sriram, and S. Sapuan, "Enhancing vocal imitations in children with autism using the IBM speech viewer," Autism, vol. 3, no. 2, 1999. [Online]. Available: https://doi.org/10.1177/ 1362361399003002004

[10] C. T. Tan, A. Johnston, K. J. Ballard, S. Ferguson, and D. Perera, "sPeAK-MAN: towards popular gameplay for speech therapy," in 9 ${ }^{\text {th }}$ Australasian Conference on Interactive Entertainment, Melbourne, Australia, 2013, pp. 28:1-28:4.

[11] M. A. Garcia and P. C. Santana, "Towards a usable serious game app to support children's language therapy," in IX Latin American Conference on Human Computer Interaction, Panama City, Panama, 2019, pp. 1-4.

[12] J. Duval and et al., "Spokelt: Building a mobile speech therapy experience," in $20^{\text {th }}$ International Conference on Human - Computer Interaction with Mobile Devices and Services, Barcelona, Spain, 2018, pp. 50:1-50:12.

[13] C. T. Tan, A. Johnston, A. Bluff, S. Ferguson, and K. J. Ballard, "Speech invaders and yak-man: Retrogames for speech therapy," in SIGGRAPH Asia 2014 Mobile Graphics and Interactive Applications, Shenzhen, China, 2014, pp. 24:1-24:1.

[14] C. O'Bryan, A. Parvez, and D. Pawluk, "An interactive play mat for deaf-blind infants," in $14^{\text {th }}$ International ACM SIGACCESS Conference on Computers and Accessibility, Boulder, Colorado, USA, 2012, pp. 239-240.

[15] H. C. Wauck, "Game features and individual differences: What makes a spatial skill training video game effective?" in $22^{\text {nd }}$ International Conference on Intelligent User Interfaces Companion, Limassol, Cyprus, 2017, pp. 197-200.

[16] E. Albassam and H. Gomaa, "Applying software product lines to multiplatform video games," in $3^{\text {rd }}$ International Workshop on Games and Software Engineering: Engineering Computer Games to Enable Positive, Progressive Change (GAS), San Francisco, CA, USA, 2013, pp. $1-7$.

[17] L. M. Nascimento, E. Santana, and S. R. de Lemos, "A case study in software product lines - the case of the mobile game domain," in $34^{\text {th }}$ Euromicro Conference, Parma, Italy, 2008, pp. 43-50.

[18] B. H. Neto, L. A. Fernandes, C. M. Lima, and J. Moreira, "Developing digital games through software reuse," Journal of Information Processing Systems, vol. 6, no. 2, June 2010. [Online]. Available: https://doi.org/10.3745/JIPS.2010.6.2.219

[19] J. Soriano, I. Pérez, and A. B. Domínguez, “Evaluación del uso de estrategias sintácticas en lectura por alumnos sordos con y sin implante coclear," Revista de Logopedia, Foniatría y Audiología, vol. 26, no. 2, Abril 2006. [Online]. Available: https://doi.org/10.1016/S0214-4603(06)70105-X

[20] D. Pollack, D. M. Goldberg, and N. Caleffe, Educational audiology for the limited-hearing infant and preschooler: An Auditory-Verbal Program, 2nd ed. Charlottesville, USA: Charles C. Thomas Publisher, 1997.

[21] M. F. Hinojosa, "Procesos de adquisición de la lengua escrita en población infantil con pérdida auditiva," Ph.D. dissertation, Universidad Complutense de Madrid, Madrid, Spain, 2016.

[22] K. Pohl, G. Böckle, and F. J. van Der Linden, Software Product Line Engineering: Foundations, Principles and Techniques. New York, USA: Springer-Verlag, 2005.

[23] P. Clements and L. M. Northrop, Software Product Lines: Practices and Patterns, 1st ed. Boston, USA: Addison-Wesley Professional, 2001.

[24] S. Apel, D. Batory, C. Kästner, and G. Saake, Feature-Oriented Software Product Lines: Concepts and Implementation. Berlin, Germany: Springer Science \& Business Media, 2013.

[25] T. Berger and et al., "What is a feature?: A qualitative study of features in industrial software product lines," in $19^{\text {th }}$ International Conference on Software Product Line, New York, NY, USA, 2015, pp. $16-25$.

[26] J. White and et al., "Automated diagnosis of feature model configurations," Journal of Systems and Software, vol. 83, no. 7, July 2010. [Online]. Available: https://doi.org/10.1016/j.jss.2010.02.017

[27] J. Nielsen, Usability Engineering. San Francisco, CA, USA: Morgan Kaufmann Publishers Inc., 1993.

[28] K. Pernice and J. Nielsen, “Usability guidelines for accessible web design based on usability studies with people using assistive technology," Nielsen Norman Group, Fremont, CA, USA, Tech. Rep., 2001.

[29] (2005) Introduction to Model/View/ViewModel pattern for building WPF apps. Microsoft. Accessed Oct. 2019. [Online]. Available: https://bit.ly/3cK9Mjl

[30] p5*js. Accessed Oct. 2019. [Online]. Available: https://bit.ly/30lM2PG

[31] M. Sierra, M. C. Pabón, L. Rincón, A. Navarro, and D. Linares, “A comparative analysis of game engines to develop core assets for a software product line of mini-games," in Reuse in the Big Data Era, Cham, Germany, 2019, pp. 64-74.

[32] M. Sierra, J. C. Martínez, G. Alvarez, and D. Linares, “Un videojuego para apoyar la terapia del lenguaje en niños con discapacidad auditiva. el caso de la descripción dinámica," in CoTH 2019 Workshop on Computing and Technology in Health, Cali, Colombia, 2019.

[33] I. do Carmo, J. D. McGregor, and E. Santana, "Strategies for testing products in software product lines," SIGSOFT Softw. Eng. Notes, vol. 37, no. 6, November 2012. [Online]. Available: https: //doi.org/10.1145/2382756.2382783

[34] A. Tevanlinna, J. Taina, and R. Kauppinen, "Product family testing: A survey," SIGSOFT Softw. Eng. Notes, vol. 29, no. 2, March 2004. [Online]. Available: https://doi.org/10.1145/979743.979766

[35] H. Hartmann, F. van der Linden, and J. Bosch, "Risk based testing for software product line engineering," in $18^{\text {th }}$ International Software Product Line Conference, Florence, Italy, 2014, p. 227-231. 